\title{
LA VISIÓN DE GÉNERO Y EL ESPACIO INTELIGENTE
}

\author{
Carlos Hernández PeZzi \\ Universidad de Málaga
}

Recibido 09/02/2011

Aceptado 20/05/2011

\section{Resumen}

El ensayo traza una concepción, $1^{\circ}$ del espacio europeo como un espacio de derechos y deberes, $2^{\circ}$ del espacio físico y virtual como recipiente accesible y poroso y $3^{\circ}$ del espacio inteligente como superación de las tres brechas digitales, del conocimiento, el uso, y el acceso. La influencia recíproca de nuevos modelos sociales y de la web 3.0, a través de las redes sociales que transforman el modelo físico de la ciudad. Se busca un espacio público entendido como resultado de esas tres primeras concepciones y del espacio inteligente como aquel que además de igualitario es accesible sin brechas, como fundamento y objetivo de la ciudad interactiva. La ciudad digital como resultado de la interacción de espacios públicos y privados inteligentes, no discriminatorios para un nuevo modelo social de plena ocupación del espacio real y virtual por las mujeres.

Palabras clave: Género, espacio europeo inteligente, modelos y redes sociales, web 3.0, brechas de conocimiento.

Feminismo/s 17, junio 2011, pp. 65-89 


\begin{abstract}
Of «the vision of gender and the intelligent space» test trace a conception, 1 of the European space as an area of rights and duties, $2^{\circ}$ of the physical and virtual space as accessible and porous container and 3rd grade of intelligent space as overcoming the three digital gaps of knowledge, the use and access. The reciprocal influence of new social models and web 3.0, through the social networks that transformed the physical model of the city. You are looking for a public space as a result of these first three conceptions and the intelligent space as one who in addition to equal is accessible without gaps, as rationale and objective of the interactive city. The digital city as a result of the interaction of public and private spaces intelligent, non-discriminatory for a new social model for full occupation of the real and virtual space for women.
\end{abstract}

Keywords: Gender, intelligent, notions of space, social models, social models of networks, web 3.0, gaps of knowledge. 


\section{Europa y las ciudades}

La visión de género ha ido ocupando los espacios del conocimiento, de la ciencia, la tecnología y las ciencias sociales. El espacio urbano y el espacio íntimo, o privado, se han revisado a la luz de lo que sabemos sobre el uso y la percepción de esa construcción multidimensional cuya percepción y relación con el tiempo cambia constantemente. Arquitectura y planeamiento urbanístico se han venido realizando bajo una nueva concepción, que se ha ampliado a documentos presupuestarios y estudios económico-financieros que han ido sumando posiciones de racionalización de los paradigmas de igualdad en al acceso y uso de la ciudad.

Este Ensayo pretende establecer un proceso fluido de concepción, $1^{\circ}$ del espacio europeo como un espacio de derechos y deberes, $2^{\circ}$ del espacio físico $\mathrm{y}$ virtual como recipiente accesible y poroso y $3^{\circ}$ del espacio inteligente como el resultado de la superación de las tres brechas digitales, del conocimiento, el uso, y el acceso, abiertos sin distinciones. La consecuencia es un espacio público ${ }^{1}$ entendido como resultado de esas tres primeras concepciones y del

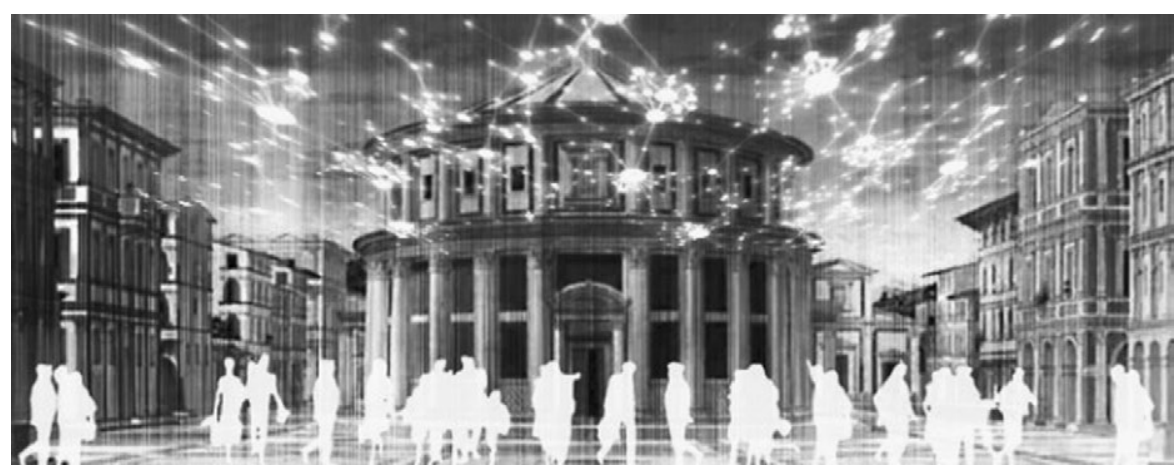

La imagen es una re-elaboración de Francesco Cingolani de la «Città ideale, Galleria nazionale delle Marche, Urbino de Pietro della Francesca».

1. INNERARITY, Daniel. «El nuevo espacio público». Espasa. Madrid. 2006.

Feminismo/s 17, junio 2011, pp. 65-89 
espacio inteligente como aquel que además de igualitario es accesible sin brechas, que debe entenderse como fundamento y objetivo de la ciudad digital. La ciudad digital como resultado de la interacción de espacios públicos y privados inteligentes y, por eso, no discriminatorios para un nuevo modelo social.

A la progresiva difusión de la Carta Europea de las Mujeres en la Ciudad, le han seguido en España la Ley Orgánica 3/2007², y el RDL 2/2008. Sin embargo, la percepción general es que la visión parcial, de alguna manera limitada, de la estructura de conocimiento de la ocupación del espacio urbano, viene legitimándose por una noción cuantitativa de los standards y requisitos urbanísticos obligatorios, más que por el resultado de la aproximación sugestiva o persuasiva de las políticas públicas de identidad igualitaria del espacio como entidad al alcance de todos los seres humanos. Contribuyen a ello ciertas visiones bienintencionadas, pero poco científicas, que reivindican legítimamente aspectos sectoriales del uso de los equipamientos, movilidades o servicios urbanos como requisitos puntuales de igualación formal sin trascender el apriorismo planificador de la separación entre el espacio público y el privado. Dicotomía acentuada por la consagración de las células de la telépólis, caracterizada unilateralmente como el espacio nuclear de la relación digital con la ciudad contemporánea. La sublimación europea de esa idea de ciudad ha sucumbido de la mano de la arruinada Estrategia de Lisboa, política que ha sido incapaz de frenar la falta de bienestar que la «sociedad del bienestar» ya no garantiza sino como un 'estado social europeo dual' de inclusión y exclusión. La defensa del «estado de bienestar europeo», sugiere que los recortes a éste en materia de reforma laboral, económico-financiera y de servicios deban, por efecto de la crisis, mantener ese esquema dual ineficiente en cuestiones básicas de acceso, empleo o uso efectivo de la ciudad.

$\mathrm{Si}$, hasta ahora, la introducción de la perspectiva de género en los instrumentos de planeamiento y planificación representaba un plus de calidad relativamente utilizado, ahora la situación cambia y se hace preceptiva. Pero la igualdad efectiva de hombres y mujeres en las políticas de suelo, en derechos urbanos peligra en el momento en que las políticas mundializadas mediante medidas anti-crisis se han centrado en el socorro del capitalismo financiero y en la precarización o acoso del «estado de bienestar» o su recorte, como si el cambio hacia un «nuevo modelo productivo» pudiera hacerse desde las mismas bases culturales y sociales anteriores a la crisis. Es discutible que las

2. Ley Orgánica 3/2007 de 22 de marzo, para la Igualdad efectiva de mujeres y hombres, art. 31.3, y el Real Decreto Legislativo 2/2008, de 20 de junio, por el que se aprueba el texto refundido de la Ley de Suelo, arts. 2.2 y 10.1 
conquistas sociales estuvieran ya equitativamente repartidas y/o garantizadas para todos los inmigrantes o nativos del modelo social europeo y para la mayoría de las mujeres. En los márgenes de la ciudad europea se encontraban ya, tras cuarenta años de experiencia, numerosas bolsas de exclusión y pobreza especialmente de los jóvenes-, bajo tensiones étnicas, religiosas o económicas, que estaban poniendo en riesgo la cohesión social anterior, siempre también bajo la discriminación de las mujeres. Desigualdad que la ciudad genérica no ha conseguido reducir o enmascarar. Los suburbios y las áreas metropolitanas se salen, así pues, del discurso políticamente correcto de la ciudad europea, como emblema a identificar y reproducir, y del espacio público europeo definido como perfil categórico por su acceso y disfrute democrático.

La precipitación de la crisis y las medidas anti-crisis ha procurado el olvido de que el «estado de bienestar» entendido como «isla» en la globalización tenía ya necesidad de reformas urgentes e inaplazables, especialmente en la ciudad y en lo que hace a la plena incorporación de las mujeres al modelo productivo. El mundo ha cambiado mucho en el período final de siglo para mantener intocado un modelo que hacía agua por el desarrollo insostenible, la tendencia al envejecimiento, la ampliación a la Unión Europea de 27 miembros, las adhesiones de Turquía y otros países y el empuje irreversible de los movimientos migratorios y las economías emergentes. Europa está afronta la ineludible reforma del modelo productivo disimulando la imprescindible reforma del modelo social y del modelo urbano que esta profunda crisis lleva indisolublemente aparejada, plagada de nuevas desigualdades endógenas.

Las economías europeas salen de la crisis sin revisar - para bien o para mal -sus modelos sociales y urbanos, pese a que estos dan muestras de creciente agotamiento. Las ciudades neoliberales son como marcas que, bajo la máscara del bienestar, esconden formas nuevas de la desigualdad e injusticia, disfrazando los efectos de la exclusión de grandes sectores de mujeres y jóvenes e inmigrantes del pleno derecho a la ciudad.

Igual que ocurre con la energía, las incongruencias del modelo europeo, -la falta de cohesión social post-Lisboa-, muestran el fracaso ratificado por el desapego de los ciudadanos e implican, además, una demora en la construcción del espacio digital de redes y conocimiento que no se ha producido. Además, el doble discurso estatal y transversal europeo se salta a menudo el discurso sobre la ciudad. El énfasis en la vivienda y la poca influencia relativa de todo aquel espacio que no es público o privado enteramente, sino un espacio de mezcla, por ser comunitario, colectivo o de transición, han dejado el protagonismo a la dualidad vivienda-estado que tiene al hogar digital como el nodo de una red de espacios interconectados sin continuidad espacial en la 
realidad física de la ciudad. Esta red de espacios accesibles solo a partes de la ciudadanía puede ser abierta o cerrada, pero en sí misma constituye un espacio de relación física que está vinculado a las redes sociales de convivencia y cohesión que fundamentan un tejido urbano sano, saludable, o si se quiere, sostenible. La lentitud en europea de las estrategias informacionales y comunicacionales ha ralentizado la imparable maduración de la ciudad digital de redes y capas.

\section{La ciudad digital}

La progresiva implantación de las redes de telecomunicación y las redes sociales ha ido demostrando cómo la riqueza de contactos virtuales podía superar en complejidad, dinamismo y poder de comunicación a los de los espacios convencionalmente destinados a esa función en el espacio real. Esta implantación/suplantación espacial ha traído consigo la doble superchería de que $1^{\circ}$, la ciudad digital ya era una realidad física y que $2^{\circ}$, ya vivíamos en ella con igualdad de derechos y accesos. Por el contrario, la persistencia de las desigualdades de acceso, las brechas digitales y las censuras o zonas limitadas en la red, han dimensionado también de forma desigual el espacio digital, como espacio comunitario de alto nivel de calidad.

La muerte de William Mitchell en 2010 nos privó de uno de los principales teóricos de la ciudad digital real virtual conformada por el espacio público digital, entendido éste como ámbito de soberanía ciudadana. En la Milla Digital de Zaragoza y entre la wikiplaza de Sevilla y la red global, la conexión y interdependencia entre ciudades reales y virtuales, anticipa un cambio de atributos y escenarios e implica una transferencia de conocimiento sobre el espacio y sus nuevas dimensiones. Se anticipa así la nueva concepción del espacio igualitario como espacio público, o «espacio común múltiple» de la ciudad del futuro. Ya no imaginamos las urbes emergentes sin la hibridación de conexiones, sin la complejidad espacial de lo real y lo virtual entrelazados y sin la afloración de redes de información, tareas y personas interconectadas en un territorio público de acceso con la mera limitación de preservar de la intimidad y privacidad de la nueva ciudadanía. La ciudadanía emergente, por otro lado, tiene nuevos protagonistas suprapersonales, considerados también como «individualidades colectivas», en la medida en que están personalizadas, perfiladas e identificadas individualmente, pero también trenzadas, abiertas y agrupadas según sus propias pautas de cooperación, solidaridad e intercambio. Sin pausa estamos abriendo constantemente las puertas a una nueva ciudad con componentes tomados de metáforas tecnológicas de las redes globales e iluminando la realidad urbana con nuevas luces. El espacio digital y lo 


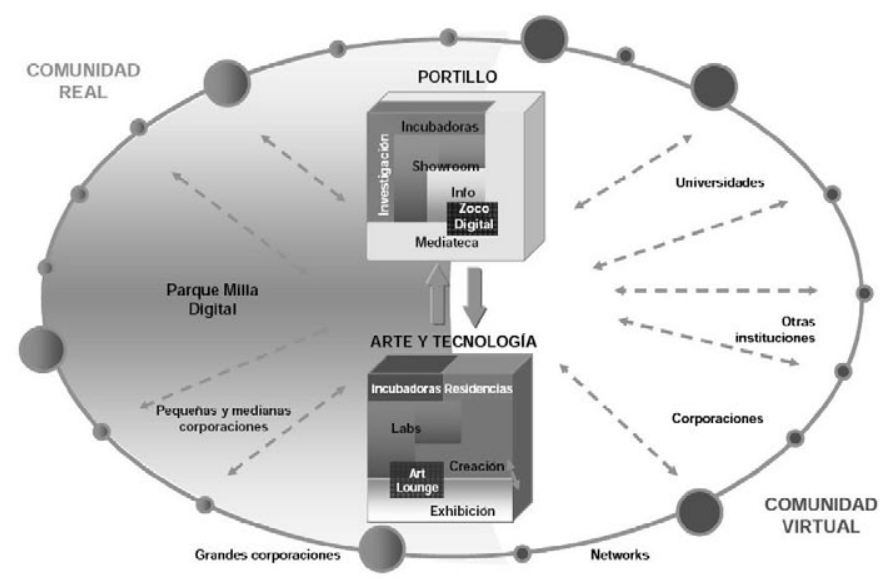

Ayuntamiento de Zaragoza Milla Digital

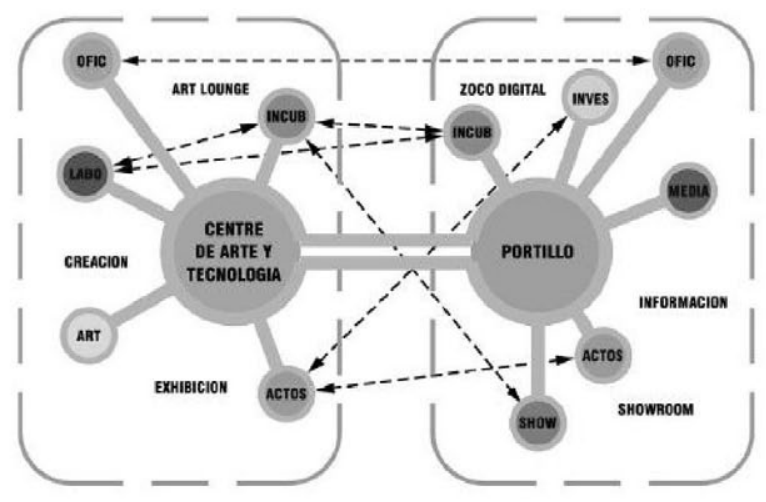

Ayuntamiento de Zaragoza Milla Digital

que se anticipa de la web $3.0^{3}$ se ha abierto como el territorio expandido, aún inexplorado del todo, que abarca los territorios de unas dimensiones casi ilimitadas entre lo real y lo virtual. La visión de género se extiende a medida que se vuelven también más y más interactivos, interdependientes y accesibles,

3. SABAdell ARTiga, Lluís. Web 3.0 y la trasformación del espacio (físico) en la web de Ecosistema Urbano. 22 de Junio de 2010. Lluis Artiga es artista y comisario especialiado en Arte y Naturaleza. Director de Post-Oil Cities y de Hibrids 2.0 aplica el concepto de Web 3.0 a los talleres expandidos on-line.

Feminismo/s 17, junio 2011, pp. 65-89 


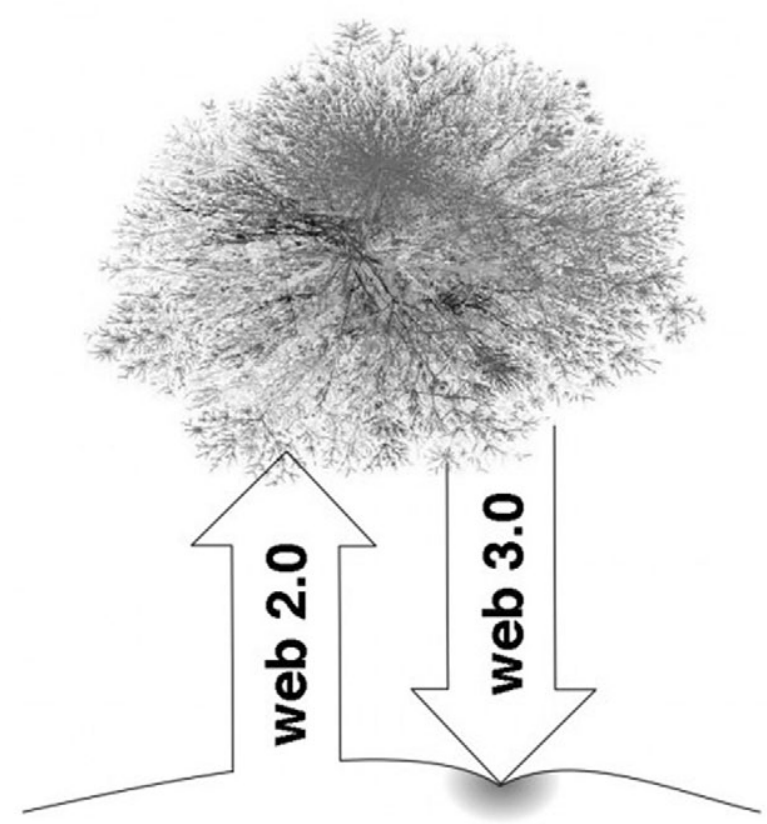

Web 3.0 y la trasformación del espacio (físico) por Ecosistema Urbano. 22 de Junio de 2010. Lluís Sabadell Artiga: artista y comisario especialiado en Arte y Naturaleza. Director de Post-Oil Cities y de Hibrids 2.0 aplica el concepto de Web 3.0 a los talleres expandidos on-line.

dotándose de una flexibilidad que no tiene hasta ahora la concepción, la construcción social del espacio, o su diseño en la práctica arquitectónica y urbana, por muy correcta que sea. De hecho, la perspectiva de género ha abierto nuevas posibilidades de acercamiento a la realidad global de los nuevos sujetos sociales, en la medida en que la incorporación plena de la mujer al sistema productivo es una incorporación plena a un nuevo modelo de ciudad. Podemos afirmar, sin lugar a dudas, que el cambio el modelo productivo que empieza a emerger en 2010, desde la crisis global, producirá un nuevo modelo social y, en consecuencia un nuevo modelo de ciudad, por la acumulación de factores de innovación disruptiva que la crisis ha congregado. Innovación que rompe los moldes y costuras de la idea de ciudad neo-liberal amable y cuidadora; idea fracasada tanto para tanto para la esfera de la «ética del cuidado» (aunque se discuta desde el feminismo) como frente a la esfera del poder. El estallido de la burbuja inmobiliaria y sus secuelas urbanas de espacios inhóspitos, inaccesibles o degradados crecen sobre la ruina hipotecaria y el desempleo. Un modelo de interculturalidad y de carácter intergeneracional es, por 
eso, la única esperanza para el cambio social del nuevo modelo productivo. No sólo de las condiciones de creación y reparto de la riqueza, de sus formas de acumulación, sino de la conformación del proyecto de un nuevo espacio público más justo y cualificado, ocupado por nuevos sujetos sociales.

La repercusión de este proceso social sobre las ciudades es clave, pues en las ciudades es dónde más fuertemente se ha producido el proceso de decadencia de la ciudad neoliberal que está en el horizonte conservador, como si nada hubiera pasado. En ese sistema, lo que el urbanista David Harvey ha denominado «acumulación por desposesión $»^{4}$ se ha venido ejecutando en las ciudades. En España y algún otro sitio más, la desposesión de las nuevas clases medias y los grupos emergentes se ha producido gracias a la acumulación de activos - vía inmobiliaria - por unos pocos, bajo el engaño a los consumidores y el consentimiento social de las sucesivas burbujas en los precios de la vivienda y en el trasvase de rentas del suelo a las sociedades de la Bolsa (y al bolsillo de muchos especuladores). En tanto algunos patriarcas han considerado las políticas de igualdad como adornos superfluos, la ciudad neoliberal ha propiciado el expolio de las hipotecas y se ha construido a imagen y semejanza del modelo social caducado: coches, unifamiliares y burbujas, en medio de un estado de embriaguez y melancolía un consumo que se espera recuperar en cuanto salgamos de la crisis. La ciudad genérica basada en ese modelo de apropiación (pretendidamente global) del espacio público y del espacio digital, se ha construido sobre la desposesión forzada, vía hipotecaria, del espacio individual; y sobre la brecha digital en el espacio de las telecomunicaciones. Es un hecho contrastado que la «acumulación por desposesión» afecta más a las mujeres, víctimas frecuentes de su propia insolidación como sujetos de ciudadanía de pleno derecho, a la vista de violencia urbana (mal denominada «violencia doméstica») contra las mujeres. Frente a una ciudad más «femenina», paradójicamente, se procura una orientación desde la visión de género de las mujeres, que debería alumbrar el nuevo modelo post-crisis y facilitar empatías e hibridación entre las redes sociales y las redes urbanas, desbordando la mera perspectiva de género, con la plena ocupación social del espacio real y digital por las mujeres ahora protagonistas frente a los actores ejecutivos de la especulación financiera.

4. HaRVEY, David. «El nuevo imperialismo: acumulación por desposesión», en Pantich, Leo y Colin Leys (ed.): El Nuevo desafí Imperial: Buenos Aires: Merlin Press - Clacso. 2004, pp. 99-129. 


\section{Espacio inteligente}

Pero volvamos a la noción del género como atributo particular de la condición inteligente del espacio. Entendemos esos condicionantes de inteligencia dentro de la competencia digital, el poder de control y autonomía del sujeto al alcance de los sistemas abiertos que caracterizan el uso del espacio como contenedor cultural de emancipación. En numerosas ciencias como la sociología, la psicología o la antropología, el género es una construcción simbólica que alude al conjunto de atributos socioculturales asignados a las personas a partir del sexo biológico que convierten la diferencia sexual en una desigualdad social entre hombres y mujeres. El feminismo post-igualitario, sin embargo, discute estas visiones de identidad y diferencia como esencias universales, una de las muchas caras del problema. Según Juan Freire, en un artículo en su blog, de 2009,

Hay dos perspectivas para aproximarse al tema de la identidad digital y de Internet. Una es creer que la presencia virtual significa un peligro para la seguridad personal y, por tanto, convenir en que si un individuo no construye su identidad digital, una tercera persona puede suplantarla y pueden ocurrir hechos indeseables. La otra perspectiva es entender la construcción de la identidad en la red como una oportunidad de aprendizaje tanto personal como profesional dentro de la cultura informacional donde vivimos inmersos.

Además de esto, la identidad puede ser múltiple y suprapersonal en el espacio inteligente. Si ya las posturas de la visión de género sobre la identidad y la diferencia abren posibilidades a una reinterpretación de los problemas de la igualdad, las dicotomías entre lo analógico y lo digital y la posibilidad de multiplicar las identidades digitales abren perspectivas inéditas al aprendizaje uso y accesibilidad al espacio de la red, de la ciudad virtual y de los espacios públicos del mundo global de las redes.

Estas consideraciones sobre igualdad y diversidad, sobre identidad múltiple y multiplicación de diferencias de acceso también dejan flancos abiertos a la relación desjerarquizada entre la visión de género y el espacio. Se abre así una vía ambigua entre el espacio especialmente interactivo, múltiple y complejo (el espacio público) de las redes y el espacio privado, dando por hecho que ambos necesitan una componente singular de conocimiento cualificado, para ser apropiables libre y democráticamente, sin limitaciones; lo que sugiere la triple apuesta del sujeto por superar las brechas de desigualdad en la competencia, el control y la autonomía, así como en el uso.

El diccionario de la Real Academia Española de la lengua define la Inteligencia (del latín intellegentřa), entre otras acepciones como la «Capacidad para entender o comprender» y como la «Capacidad para resolver problemas». 


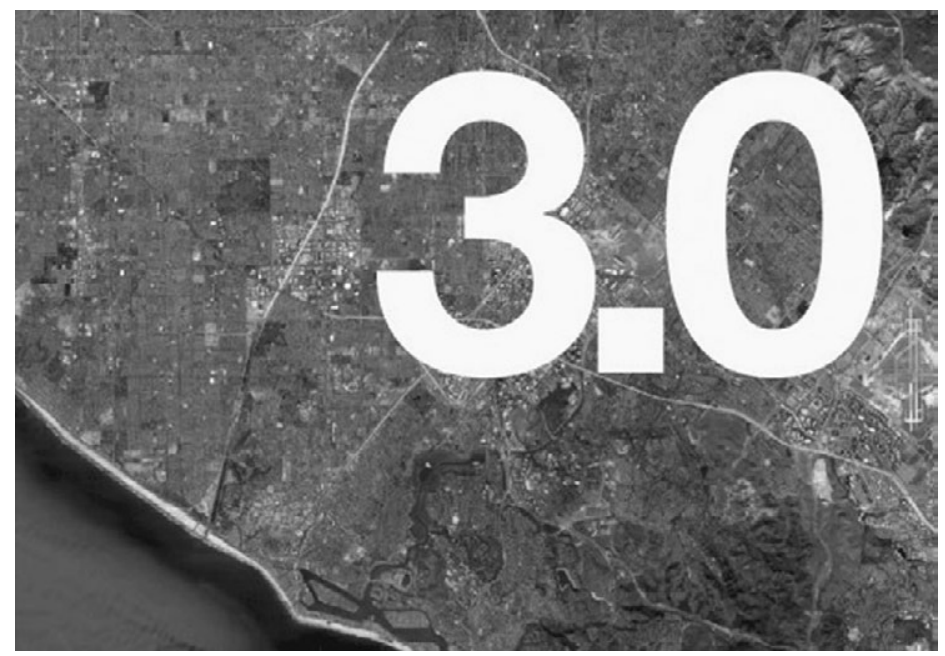

Web 3.0 y la trasformación del espacio (físico) por Ecosistema Urbano - Martes, 22 de Junio de 2010.

La inteligencia parece estar ligada a otras funciones mentales como la percepción, o capacidad de recibir dicha información, o la memoria, o capacidad de almacenarla. Nos encontramos, pues, con acepciones que encajan perfectamente con la filosofía del espacio-tiempo que está representada por la ontología, la epistemología y la naturaleza del espacio y del tiempo, tomados como variables de construcción de la ciudad; que tanto auge han tenido en el conocimiento del impacto de género sobre esta. Tanto en la comprensión, percepción o memoria, la ciudad es el lugar donde el espacio tiende a interferirse con el tiempo en conflictos de toda naturaleza. Sobre todo con el tiempo simultáneo, o con su percepción simultánea.

La aplicación científica al espacio del atributo de la inteligencia tiene un recorrido más difícil. Si la noción de ciudad es difícil de definir y precisar hoy en el conocimiento y la ciencia, la noción de espacio tiene acepciones ilimitadas de las que vamos a repasar someramente sólo dos, las que se refieren a Informática y Matemáticas, que se ajustan al objetivo de este trabajo. En informática, una breve relación de tipologías tomada de Wikipedia ${ }^{5}$, suministra

5. Wikipedia define la web 2.0 como «término asociado a un fenómeno social, basado en la interacción que se logra a partir de diferentes aplicaciones web, que facilitan el compartir información, la interoperatividad, el diseño centrado en el usuario y la colaboración en la World Wide Web.» La web 3.0 se caracteriza como un paso de cambio tecnológico (3D, 
un inventario de analogías razonables para establecer vínculos entre denominaciones de la realidad virtual y la realidad física: Espacios de color; Función de espacio constructivo Espacio duro, como variante del carácter «espacio»; Espacio afín (dotado de una aplicación). Son definiciones semejantes a las del espacio urbano: Espacio de usuario, un espacio de aplicación; Espacio de intercambio de zona (un fichero o partición); Espacio de puntuación entendido como vacío. De la codificación de caracteres pasamos al entramado de redes como un conjunto de decisiones, de complejidad y orden. Situaciones de compromiso espacio-tiempo o tiempo-memoria, comprimidas o ampliadas por la velocidad de ejecución de que disponga el usuario, de si lo comparte o lo aplica a usuarios afines.

Nociones como el espacio de usuario, o el de intercambio, o el espacio duro, sirven en esta ligera enumeración como sencillas trasposiciones de los espacios elementales de la ciudad. El conocimiento informático atribuye elementos de reflexión a la trasposición de nociones del espacio informático al espacio real. Como sostiene el arquitecto sevillano José Pérez de Lama, empezamos a vislumbrar un nuevo modelo de metáfora informática que lleve a «plantear una arquitectura que comparta las cuatro libertades del software libre: que pueda ser usada por todos; que el código fuente pueda ser leído por todos; que pueda ser modificada por sus usuarios / habitantes, y que las modificaciones puedan a volver ser distribuidas». Una metáfora arriesgada, porque el uso, lectura, reforma y distribución, seguridad, confianza o manipulación han tenido históricamente connotaciones desiguales desde el punto de vista del género.

En matemáticas y geometría el inventario de analogías es interminable. La multiplicación científica de trabajos sobre la materia, no permite entrar en complejidades científicas. El catálogo de conceptos es aplicable a la arquitectura de la ciudad, si observamos las nociones de espacios duales, separables, uniformes, etc. Un breve repaso da cuenta de su múltiple caracterización, de sus interferencias con los espacios, dimensiones y topologías de la realidad urbana: dual, vectorial, normado, multidimensional, euclídeo, compacto, para-compacto, espacio localmente anillado; espacio sobrio, espacio tangente, espacio escalado, separable, muestral, universal, uniforme..., particionado, funcional, completo, recubridor,... Aunque si nos fijamos atentamente, el espacio geométrico frente al informacional es un espacio de recintos ante el que plantear siempre el conflicto de los «dilemas de ingreso».

semántica,...), pero se piensa que, como la anterior, también dará lugar seguramente a un cambio social y una transformación física del espacio y la realidad física. 
Haremos de este sobrevuelo de definiciones un atisbo de metáfora para no caer en ninguna impostura intelectual de las denunciadas por Sokal ${ }^{6}$ y Bricmont. Desde el punto de vista epistemológico, hay que dar saltos controlados para no caer al vacío retórico, cotejando estas acepciones espaciales de la construcción del espacio social por excelencia, el espacio público; y del uso público de Internet, que se viene demandando como tal espacio público. A las redes sociales como Facebook y a las nuevas intervenciones urbanas aisladas se las está calificando ya de «islas de conocimiento»o «islas de experiencia», - en la medida en que puedan limitar el uso social de sólo algunas porciones de territorios amigables -, en lugar de abrirse a los espacios de «confines porosos» ${ }^{7}$. Confines fluidos y continuos, que son una obsesión de las ciencias sociales de la posmodernidad. Posición distinta a la de Peter Sloterdijk ${ }^{8}$ en su Trilogía Esferas. A pesar de lo que dice Rüdiger Safranski, sobre Sloterdijk, nuestra perplejidad ante el argumento aflora ante la perspectiva de que no podamos combatir un destino tan metafísico como el del enclaustradamente geométrico.

«convoca los sentidos, las sensaciones y el entendimiento para conseguir claridad sobre lo cercano. ¿Lo cercano? Lo cercano es aquello que la filosofía pasa a menudo por alto: el espacio vivido y vivenciado. Vivimos «en» espacios, esferas, atmósferas; la experiencia del espacio es la experiencia primaria del existir». ${ }^{9}$

Ya que si la experiencia de vivir transcurre en las atmósferas de la ciudad, en los microcosmos urbanos y efectivamente, en aquellos en los que lo vivenciado es lo próximo, la red rompe el límite entre lo cercano y lo global. La desenvoltura de la filosofía de Sloterdijk abre una panoplia de mundos indetectados como tales, como la compacidad del aire acondicionado, la crisis ambiental definida la espuma que encierra a los individuos en lo «redondo». En su primer volumen, el filósofo alemán habla de burbujas, en el segundo de globos y en el tercero de espumas, en una suerte de esferología plural que abarca lo redondo, aclarando que el «tercer volumen se ocupa de la catástrofe moderna del mundo redondo». Pero la preocupación geométrica por la forma, por la morfología y por la ciudad aparece cuando cita, a Hans Blumenberg, Salidas

6. SoKAL, Alan. «Más allá de las imposturas intelectuales. Ciencia, filosofía y cultura». Paidós. Transiciones. Barcelona 2009. Págs. 44-45 y siguientes.

7. En expresión de Sheila Benhabib, citada por GALLI, Carlo. «La humanidad Multicultural». Katz Editores. Madrid, 2010, pp. 22, 44 y 64.

8. SlOteRDiJK, Peter. «Esferas I, II y III» «Esferas I», «Esferas II» y «Esferas III». Editados por Ediciones Siruela S.A. Madrid, 2003, 2004 y 2006, respectivamente.

9. SAFranski, Rüdiger en el Prólogo a SLOTERdijK, Peter. «Esferas I». Madrid, 2003, pp. 13 y 14 .

Feminismo/s 17, junio 2011, pp. 65-89 
de la caverna "La ciudad es la repetición de la caverna con otros medios» ${ }^{10}$ y localizando una metafísica universal de la polis que abarca una historia de la forma «del mundo poliesférico que vivimos hoy no es ya el globo sino la espu$m a »-$ concluye $-{ }^{11}$. En ese sentido, el concepto de «techo de cristal» se habría transformado en el de «esfera de cristal», o burbuja, de la que sólo se sale si se rompe una o se pincha la otra.

Esta noción proveniente del discurso de las geometrías ${ }^{12}$ es cara a los matemáticos y atractiva para los arquitectos, pero muy excluyente si consideramos la esfera como la metáfora para definir la sociedad global y la ciudad digital, como islas y la noción de la arquitectura y el urbanismo de la «Foam City». Al fin y al cabo la posmodernidad digital en arquitectura, - por ejemplo, en Zaha Hadid -, se asocia con formas bulbosas, placentas. El espacio celular de los telepolitas urbanos se encaja en una suerte de cavernas telemáticas en las que los eremitas digitales se dedican a la meditación electrónica y a su comunicación con el mundo a través del cosmos global y esférico, lo que excluye el contacto del común de los sentidos con la vida de lo real y sus avatares. Las complejidades urbanas en el espacio contemporáneo no parecen encontrarse en esa visión de recintos aislados. La Web. 3, por el contrario se aventura en otra transformación física y dimensional de la ciudad. Sin una forma preconcebida, la ciudad-red es una metáfora sociológica. La aproximación a la geometría de Sloterdijk (tras la de Jacques Derrida a propósito de Hussserl en «Introducción a 'El origen de la geometría de Husserl ${ }^{13}$ ) se muestra excluyente, como acredita la cita del alemán al recordar la inscripción en la entrada de su Academia de la frase atribuida a Platón «que decía: manténgase alejado de este lugar quien no sea geómetra». Como casi todas las multi-dimensiones de los espacios geométricos excluyen atributos de percepción porosa y de flujos, su condición sería restrictiva o restringida. Los espacios estáticos, esenciales o universales están en decadencia, en tanto otros entran en clara sintonía o sincronía en el tiempo con los problemas que apunta el espacio inteligente de la era digital (y el espacio público de la ciudad digital). Por ejemplo el espacio abierto, aunque asume el riesgo de la identidad digital no acreditada o camuflada, es más atractivo a la creatividad y la innovación. En este sentido,

10. SlOterdijK, Peter. «Esferas I». Madrid, 2004. Página 219.

11. SLOTERDiJK, Peter (Esferas I, página 73).

12. FERNÁNDEZ, Isabel. Por primera vez, por una matemática española, Isabel Fernández, se ha presentado a nivel del Congreso Internacional de Matemáticos de Hyderabad (India 2010) un trabajo sobre las superficies de geometría variable de las pompas de jabón.

13. DERRIDA, Jacques. «Intoducción a 'El origen de la geometría de Husserl». Ediciones Manantial. Buenos Aires 2000. pp. 12, 108, 176 y 188. 
siguiendo a Husserl, la web 3.0 podría ser un ejemplo paradigmático de una «forma en formación». La simulación de un paisaje topológico, que puede hacerse en el espacio virtual casi con más realismo que en la ciudad misma, pre-configura el campo de batalla de los dilemas de ingreso, de la igualdad, la diferencia o el acceso al espacio físico. Por eso, conocer los límites de la capacidad de apropiación del espacio digital es necesario para tener la libertad de saltárselos o mantenerlos en la hipótesis auto-protectora. Tal vez como ocurre en la experiencia urbana, el anticipo o el vector de la ruta es tan importante como el itinerario a recorrer, es una apuesta más que sólo un derecho a la igualdad; resulta más inteligente ofrecer libertad segura a quienes quieren navegar en la red sin naufragar en ella.

En la nueva percepción de la ciudad el espacio con perspectiva de género se identifica como recipiente de alta calidad frente al espacio indiferente o genérico, en la medida en que la noción de inteligencia se encuentre asociada a la apropiación y soberanía del usuario sobre el espacio en su conjunto, público o privado, y a su inseparable relación con el cuerpo y con el tiempo, con los que mantiene una declinación común. Las nuevas identidades de las mujeres para ser libres requieren de ropajes diferentes -algunos los llamarían máscaras- y otros como la opaca frontera del «burka», nos llevarían abrir un frente no deseado en este contexto

El nuevo modelo social está unido a la recuperación de una noción participativa y accesible de la nueva ciudad, que puede ser la ciudad P2P (intercambio de archivos) que augura Juan Freire ${ }^{14}$ o la ciudad por capas, pero ya no parece que vaya a ser la versión física literal de la La ciudad-red de Manuel Castells; al menos si no se establecen nuevos cauces de accesibilidad, ocupación y soberanía del espacio público y consecuentemente del espacio digital por la ciudadanía, así como otra noción de los ciclos vitales y temporales globales. Eso no ocurre, porque siguen existiendo brechas de género (menos del $22 \%$ de ganancia anual y menos del $16 \%$ en salario/hora en las empresas), de participación proactiva (siempre inferior al $40 \%$ en el gobierno local de las ciudades) y de formación del capital humano (12, \% de catedráticas en las Universidades españolas y grandes diferencias en las universidades europeas y entre éstas y las asiáticas), y desigualdades en el tránsito de la formación a la producción. En uso de la ciudad la inercia de la denominada «ley» del 35/65\%

14. Freire, Juan. Ver en la web «Ciudades innovadoras y sostenibles». http://nomada.tumblr.com/post/1003243471/la-gestion-de-la-identidad-digital-unanueva habilidadhttp://dig.csail.mit.edu/breadcrumbs/node/215. 
que afecta a las diferencias entre mujeres y hombres respecto a vivienda movilidad, empleo y rentas. ${ }^{15}$

\section{El urbanismo de las redes}

La utopía neo-liberal de la ciudad competitiva y del marketing urbano ha acabado por sumirnos en la crisis más grande de la historia. Esa ciudad no se construía con ladrillos sino con hipotecas, con trasvases de rentas y con diferencias de acceso a la red. El urbanismo de la red y la era digital son, pues, inseparables de un concepto de apropiación en el que los espacios no se consiguen por hipoteca o expropiación, sino por flexibilización de los accesos, gratuidad y participación equilibrada. Es lo que se han denominado espacios porosos. En las Telépólis, el espacio de redes ha trascendido los límites de la casa y ha puesto el espacio individual de más de 500 millones de personas en las redes, Facebook, entre otras. La ciudad real de la especulación inmobiliaria ha cedido sitio a la ciudad virtual. Los nuevos modelos sociales más igualitarios desde la perspectiva de género están prefigurándose antes en los modelos urbanos alternativos, en los nuevos movimientos sociales urbanos, en los modelos avanzados de participación y en la interactividad de las redes urbanas de Zaragoza, Sevilla, Barcelona o Córdoba o Toronto, - por citar biodiversidad de modelos -, mucho más que en el modelo uniforme del pensamiento único de la ciudad competitiva, descabalgado por la crisis y el estallido de la burbuja. Y paradójicamente esos modelos osmóticos son reales, se parecen más a los de las redes sociales que a las utopías caducadas del tardo-capitalismo, puestas en la picota por su falta de democracia urbana.

La democracia deliberativa en lo local, donde funciona, está aproximándose a la formulación de ciudad como república accesible global; no como república de hogar digital independiente, como alardea muy equívocamente, la multinacional del espacio privado Ikea. Esta fórmula deliberativa converge con la democracia representativa y permite emplear nuevos métodos frente a la «ciudad indiferente», la del despilfarro, que alimenta el abuso del poder local y las prácticas corruptas de la democracia inmobiliaria. La ciudad digital se convierte en un procedimiento, que afecta a la sustancia de las cosas, a su estructura. De forma creciente, los debates urbanos se convierten así, gracias a este procedimiento participativo, en foros deliberativos que tocan la raíz misma del acceso a la democracia urbana, mediante resortes de redes antes impensables. De esta forma, lo que la democracia representativa no alcanza a reconocer, por las inercias perversas de un sistema que convierte en

15. Fuentes: Datos de varios organismos y Ministerio de Igualdad del Gobierno de España. 


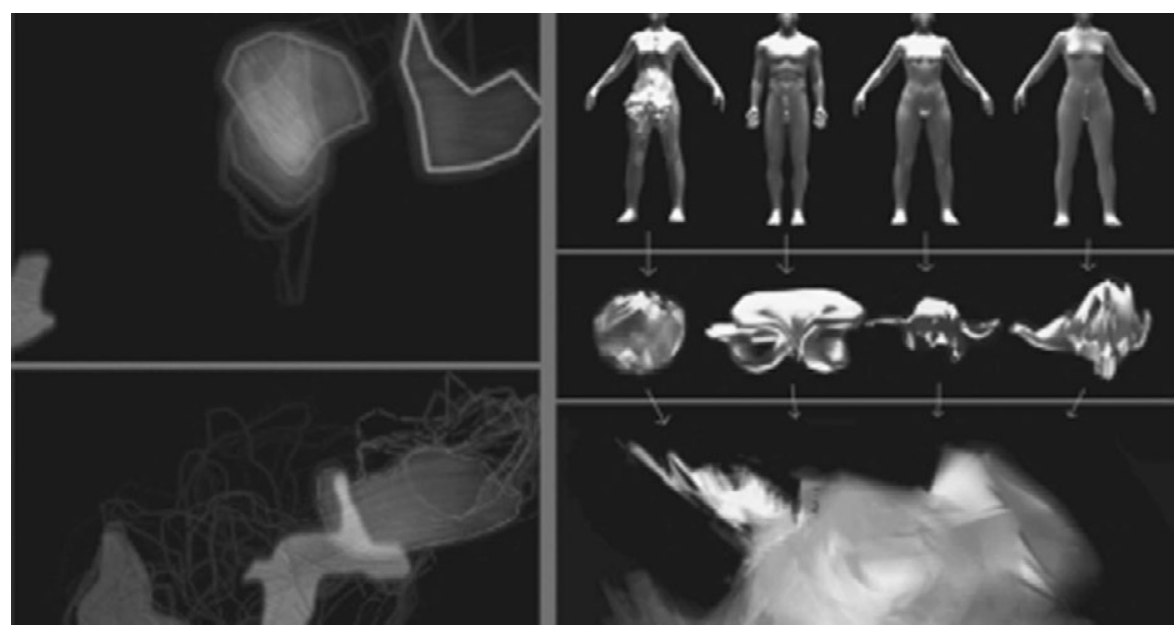

Cuerpos en red ETP: European Teleplateaus.

DESORIENTACIONES - Telemática abstracta, presencia amorfa y proximidad sin identidad - Producida por el grupo ETP-Madrid.

AMORFOGÉNESIS - Arquitectura colectiva y espacios digitales en 3D generativos no cartesianos - Producida por el grupo ETP-Praga en colaboración con Jaime del Val.

clientelares los movimientos sociales urbanos, lo alcanzan parcialmente las redes con un modelo de barrio, de vecindad, de ciudadanía. Nuevos mecanismos se alzan con un alto nivel de interactividad, que luego afecta en directo a la política y al gobierno local en lo que se puede llamar apropiadamente ciudad interactiva. ¿Puede esta ciudad de interactividad digital permanente convertir alguno de sus paradigmas en realidades urbanas? Tal vez, siguiendo la estela de esa sentencia por el cual se asegura que «la naturaleza imita al arte», la ciudad se convierta en espejo de las redes sociales; y sus colectivos más dinámicos en agentes públicos de una democracia interactiva, que combina a la vez el gobierno participativo y la deliberación en red con la representación democrática clásica.

Esta visión tendría algunas repercusiones en la construcción de la ciudad, de forma que se perderían paulatinamente las nociones centro-periferia, inclusión-exclusión, barrio-ciudad, entre otras y aparecerían nuevas convergencias colectivas a favor de una inter-territorialidad común, más representativa y global de la ciudad en su conjunto. Una visión así establecería las bases del interés general muy por encima de las particularidades desmembradas en que la óptica neoconservadora traduce los tejidos sociales, dejando siempre pospuesta la igualdad plena de hombres y mujeres en el uso del espacio. 
Frente a la extensión sobrepujada del city-marketing, ${ }^{16}$ como valor de valores refundidos en una marca, la asimilación consecuente del discurso de igualdad de género es un indicador de valor que puede hacerse visible y parametrizarse con relativa eficiencia, como ha sucedido con el reconocimiento de buenas prácticas en esta materia a Gijón y otras ciudades.

La ciudad digital debería ser el horizonte de una ciudad igualitaria de redes interconectadas, que tuviera en cuenta los grandes cambios del principio de siglo. Los cambios de los consumidores a los prosumidores (prosumers) aflorarán seres urbanos productores y consumidores de energía e información a la vez, distintos de los del siglo XX. Los cambios en la esfera de lo virtual, luego pasarán a la realidad material en forma de espacios mucho más fluidos, accesibles y soberanos de los actuales. Es fácil avanzar que el paso de las mujeres consumidoras, que ejercen hoy el poder de decisión sobre el mayor porcentaje de renta familiar de gasto evolucionará hacia mujeres prosumers productoras y consumidoras de bienes y servicios, información y conocimiento; cambio que se reflejará pronto en una apropiación de los espacios necesarios para adoptar el nuevo rol.

\section{La ciudad interactiva}

En esa ciudad «la visión de género y el espacio inteligente» estarán íntimamente hibridados de forma que sean coherentes con los nuevos papeles, no sólo porque el género ha invadido positivamente el escenario de las nuevas tecnologías, sino porque "género y TICs» son realidades de tipo igualitario que van acabando paulatinamente fragmentación del espacio urbano, en función de la identidad y de la condición digital, caracterizando definitivamente el espacio público como catalizador de cohesión social y colectividades locales, es decir, aunando individualidad colectiva y personalidades grupales, desde lo inter-generacional y lo intercultural como bases de una nueva mutación de los objetivos del género entendido como atributo del espacio inteligente, compartido y cooperante de la ciudad de nuestro tiempo.

La sensibilización por el enfoque de género en la construcción del espacio físico y la construcción del espacio social ha hecho que los espacios de la ciudad se vayan estudiando de otra manera. La identidad digital, las redes sociales y el uso de las nuevas tecnologías nos permitirán caracterizar el espacio público como el lugar de libre expresión por excelencia. De hecho el lugar es como el sitio digital, suele enterrar arqueologías narrativas durante tiempos

16. Puig, Toni. «Marca Ciudad. Cómo rediseñarla para asegurar un futuro espléndido para todos». Paidós. Barcelona. 2009. pp. 253, 271.

Feminismo/s 17, junio 2011, pp. 65-89 


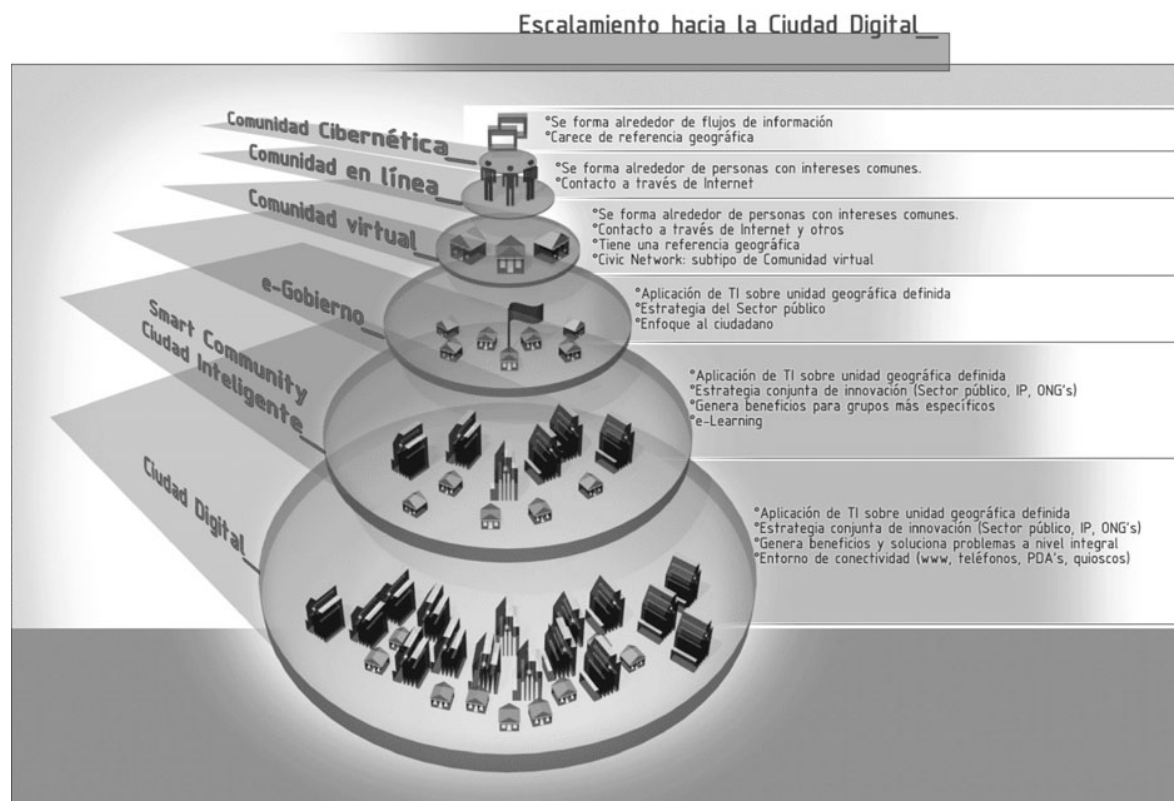

Infotec Conacyt-Fideicomiso México 2004. CIUDADES DIGITALES. Página 9. www.cibersociedad.net/public/documents/45_epi6.pdf

ilimitados. Como recuerda J. Pérez de Lama ${ }^{17}$ a propósito de la «wikiplaza» si los lugares hablan de nosotros es posible que como consecuencia volvamos a interesarnos por las características (calidades) físicas de nuestro entorno. Por muy contingente que sea el ius publicum europaeum que alberga entidades suprapersonales, en una Europa entendida como espacio público por antonomasia, los de más calidad serán los que contengan capacidades de memoria y percepción y no en los de consumo, mediocres o degradados, o desgarrados.

Por eso la vinculación de espacio público con identidad digital, depende de reglas de juego limpio a la hora de compartir y acceder a las redes, con seguridad, confianza y nuevas posibilidades de entretejer lazos de conocimiento. No otra cosa es el objetivo perseguido por la universalidad de la red, pero los riesgos son evidentes. Sin un espacio comunitario de calidad no hay un espacio público utilizable y la red ha de favorecer una transparencia que produzca confianza, si no quiere que se transmuten las identidades digitales

17. PÉREZ De Lama, José. http://www.hackitectura.net/.

Feminismo/s 17, junio 2011, pp. 65-89 


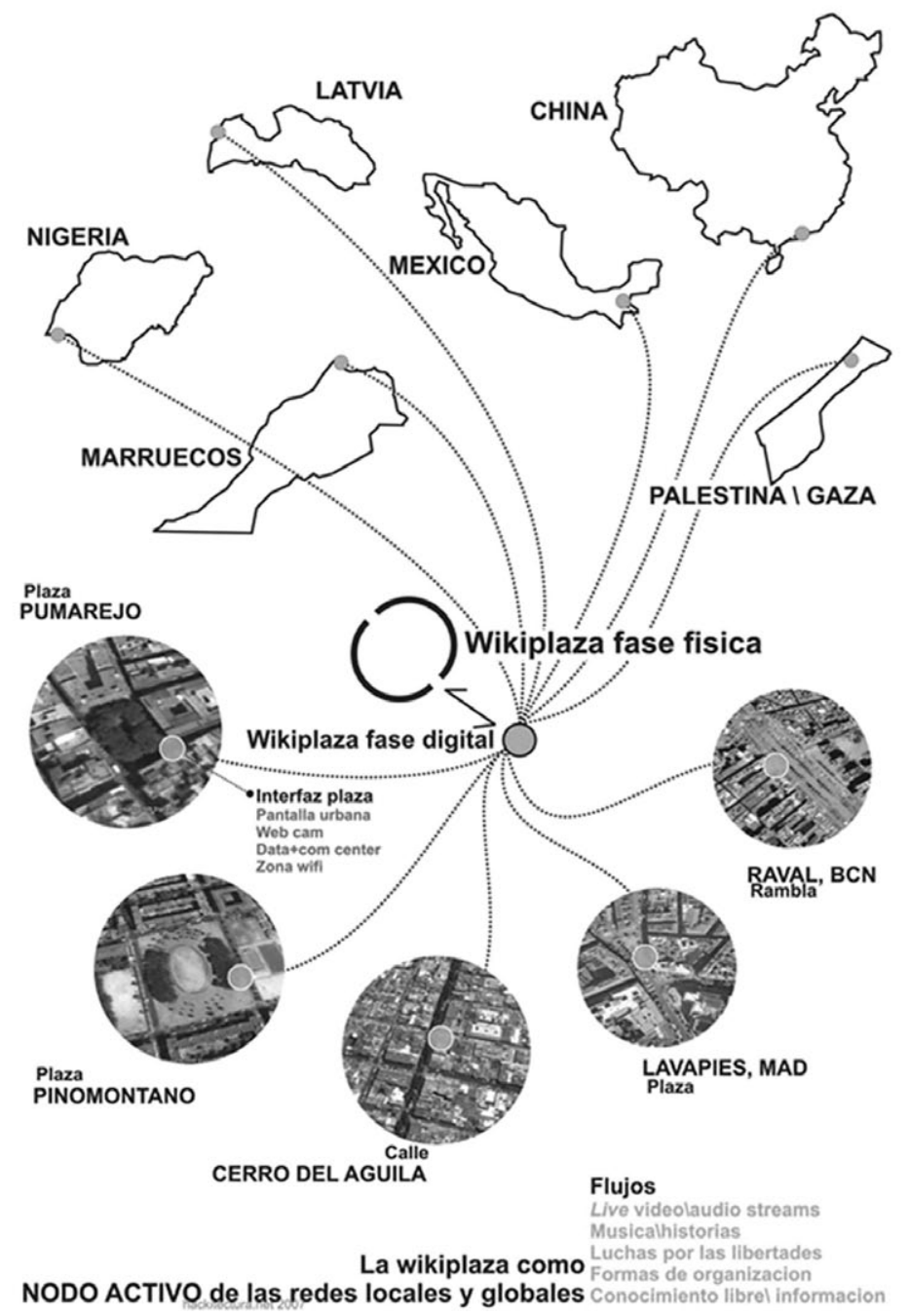

Wikiplaza

PÉREZ DE LAMA, José. http://www.hackitectura.net/

en máscaras utilitaristas y los escenarios públicos en artificios teatralizados o temáticos. El concepto de «vecino», «usuario» o «amigo» extrapolado de la sociedad a las redes, tal como ha sido transformado o manipulado por estas y así devuelto a la red, permite tanto crear o romper fronteras de comunicación. Tiene un carácter ambivalente que articula redes de metáforas y simulaciones, 
lo que lleva también a la impostación del simulacro de la realidad y al espectáculo de una comunicación tergiversada según los papeles que ejerzan sus protagonistas. El proyecto de espacio público digital implica nuevas relaciones de construcción social del espacio y del sujeto en relación con este. ${ }^{18}$ La ciudad interactiva requiere un nuevo tipo de sujeto urbano, pero las redes sociales no deben jugar a los SIMs. Se trata de que las relaciones entre el Yo y el Otro sean relaciones de cercanía/lejanía que permitan interactuar sin suplantar las identidades digitales de los sitios y las personas, acrecentando las posibilidades de conocimiento e interrelación y comunicación local o a distancia en las redes urbanas a través de confines porosos, no difuminados, o simulados por un falso universalismo de la esencia de la igualdad o de la diferencia unívocos, como intentos de ontologías del ser contemporáneo ya caducados en su versión universalista.

En la ciudad interactiva, la visión de género ha de ocupar democráticamente la igualdad en el desarrollo local sostenible y el urbanismo mediante la construcción social de un espacio inteligente que propicie la ocupación plena del espacio público de las ciudades y de las redes por parte de las mujeres. Espacios que cuenten con los atributos de igualdad en el uso, acceso, razón, belleza y vida, entre otros (protección, seguridad,...). ${ }^{19}$ Por extensión podríamos ampliarlos a aquellos de la ciudad que cumplen similares requisitos. Mediante las redes, y el urbanismo 3.0 la ciudad digital se ha de volver más participativa y democrática y la ciudad real más entretejida y solidaria, en suma, una ciudad interactiva de espacios físicos y virtuales inteligentes.

\section{Valores e indicadores}

Pero no nos dejemos llevar de discursos seráficos porque no estamos ante una tendencia asentada, ni mucho menos. No es muy consolador pasar del «techo de cristal» a la «esfera de cristal». Tampoco poner la esperanza en la transformación física del urbanismo realmente existente. No es fácil el cambio, ni está a la vuelta de la esquina de la salida de la crisis. El urbanismo actual

18. Según datos contrastados de la OIT, la mujer está ocupando progresivamente el espacio de la red en todo el mundo hasta entrar en condiciones de igualdad en los países desarrollados (50\%) y acercándose, por pura necesidad, al $45 \%$ en los países pobres, está construyendo el espacio igualitario, pero falta transmitir la condición que ya no es de dualidad sino de porosidad y atributo del espacio inteligente.

19. Por definición adversa, caracterizamos los espacios de uso limitado, con acceso precario o restringido, irracionales, feos y/o inhabitables, como aquellos resultantes del consumo o de la mediocridad, que tanto han crecido al amparo de la burbuja inmobiliaria y la mercantilización del espacio.

Feminismo/s 17, junio 2011, pp. 65-89 
construye burbujas. El futuro, en cambio, se construye con formas complejas. Los urbanistas hacemos todavía geometría bidimensional, poco asimilable a la lámina de las superficies alabeadas de las pompas y las burbujas y poco entretejida con las redes y las nuevas redes sociales. En este sentido, la construcción de la web 3.0 es una «forma en formación», la ciudad es una forma en contradicción, entre muchas razones porque la geometría es variable en la red y anárquica en la ciudad.

El discurso avanzado en esta materia por Freire y Gutiérrez-Rubí, ${ }^{20}$ entre otros, aporta información sobre las orientaciones de los procesos que obligan a cambiar el discurso de los urbanistas en la web 2.0. A la vez, introducen la perentoria necesidad de innovar en los foros universitarios y científicos en materia de visión de género, revisando valores, y mejorando los mecanismos de establecer indicadores de evaluación. En plena adaptación a su creciente aceptación social, ambas cuestiones están en proceso de cambio urgente y de puesta al día desde el punto de vista de la construcción del nuevo espacio urbano inteligente que se desarrollará tras la crisis; un discurso muy desconectado de las sinergias que hay que provocar tras pinchar las burbujas y revisar las nociones de acceso e igualdad reconociendo el nuevo escenario. Basta con enunciar los problemas de la nueva visión sobre el cuerpo, la noción de los sujetos como «nativos» e «inmigrantes» digitales y sus consecuencias aplicadas a niñas y mujeres o el revolucionario proceso de aprendizaje de las nuevas tecnologías y las redes, que hace aprender, - de forma inversa a como ha sucedido históricamente -, a las madres de las hijas, y a las abuelas de las nietas y de las madres. El síndrome de Ulises, la inmigración digital y el aprendizaje inverso empiezan a ser obligado patrimonio también de las mujeres Por eso hay que elaborar un discurso basado en la investigación científica del cambio de marco y un diálogo estable sobre la formación de la forma en la ciudad y en la red bajo los parámetros nuevos.

También la formación social en perspectiva de género tiene sus altibajos en cuanto trata con la red. Si Castells ponía en tela de juicio la noción de ciclos vitales y temporales en su clásico "La ciudad-red», cuestión que ahora habría que elucidar con un nuevo discurso del cuerpo y del espacio-tiempo. Los ciclos son la raíz de la vida. La web 3.0 se constituye mediante procesos participativos abiertos en redes cercano/lejano y apuntan a ciclos cotidianos o globales. A estos efectos, la ciudad no debería construirse en recintos estancos. La curva no es la forma contemporánea estereotipada de la ciudad o

20. Freire, Juan. Gutiérrez-Rubí, Antoni. Prólogo de Pisani, Francis «2010-2020. 32 Tendencias de cambio» Septiembre 2010. http: //www.gutierrez-rubi.es/wp-content/ uploads/2010/09/32Tendencias de cambio.pdf 


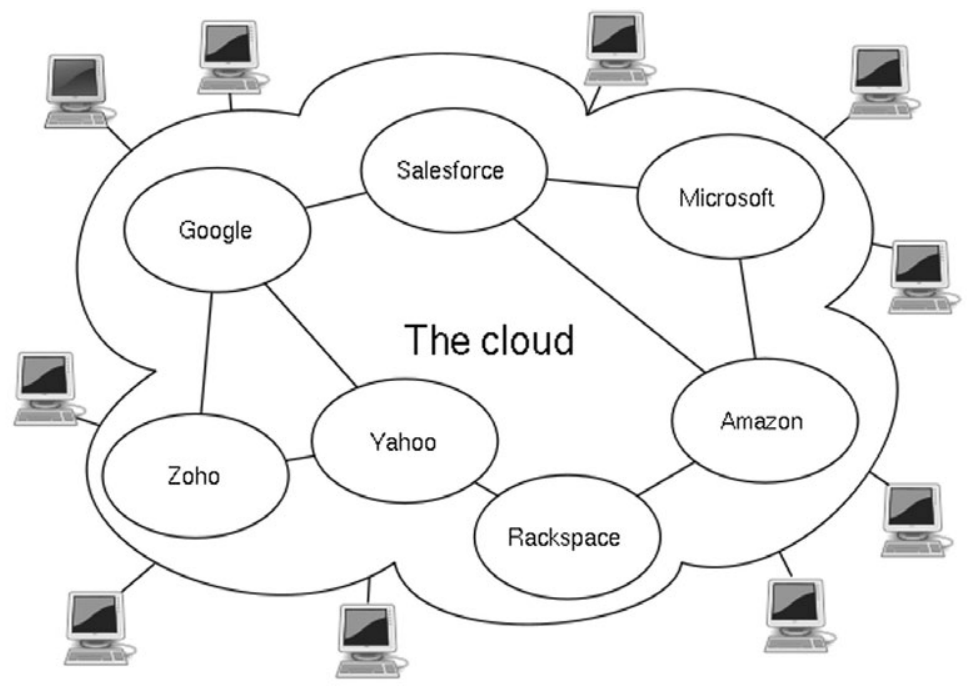

Wikimedia Commons Wikipedia

de la arquitectura... como la esfera no lo es de la red. Las multi-dimensiones son la componente esencial del proyecto de la red y de las ciudades cuando aseguran modelizaciones de proyectos compartidos. El alabeo de la realidad abarca superficies geométricas impuras y, como tales, utiliza verdades contrapuestas, contingentes, precarias. Ese es el desgarramiento de la modernidad que afecta al tiempo que vivimos. Pero no se puede negar que las metáforas de la red -como la visión informática de «cloud computing $»^{21}-$, por ejemplo,

21. Wikipedia. En este tipo de computación todo lo que puede ofrecer un sistema informático se ofrece como servicio, ${ }^{1}$ de modo que los usuarios puedan acceder a los servicios disponibles «en la nube de Internet» ${ }^{2} \sin$ conocimientos (o, al menos sin ser expertos) en la gestión de los recursos que usan. ${ }^{3}$ Según el IEEE Computer Society, es un paradigma en el que la información se almacena de manera permanente en servidores en Internet y se envía a cachés temporales de cliente, lo que incluye equipos de escritorio, centros de ocio, portátiles, etc. Esto se debe a que, pese a que las capacidades de los PC han mejorado sustancialmente, gran parte de su potencia se desaprovecha, al ser máquinas de propósito general. Cloud computing es un nuevo modelo de prestación de servicios de negocio y tecnología, que permite al usuario acceder a un catálogo de servicios estandarizados y responder a las necesidades de su negocio, de forma flexible y adaptativa, en caso de demandas no previsibles o de picos de trabajo, pagando únicamente por el consumo efectuado. El concepto de la computación en la nube empezó en proveedores de servicio de Internet a gran escala, como Google, Amazon AWS y otros que construyeron su propia infraestructura.

Feminismo/s 17, junio 2011, pp. 65-89 
coinciden con la aspiración a abrir sucesivos «confines porosos» de espacios de certeza por muy contingentes o fragmentarios que sean. Las geometrías como conjunto de verdades fragmentarias lo son, en la red y en la ciudad, tanto como en la arquitectura, pero no pueden poner límites a la humanidad que se reconoce en un nuevo imaginario colectivo, ni convertirse en fines en sí mismos, en el espacio ciudadano abierto.

El cambio de paradigmas urbanos se asentará sobre la inteligencia que se demuestra en los valores de igualdad y democracia urbana, pero bajar de las nubes informacionales a las ciudades reales requerirá de una vuelta de tuerca a los discursos sobre el género y sobre la ciudad, para que converjan e interactúen en la dirección adecuada a los cambios que vienen.

\section{Referencias Bibliográficas}

Agrest, Diana. Conway, Patricia. KAnes Wisman, Leslie. Editors. «The sex of architecture». N.Y. Harry N. Abrams, Inc. 1996.

BETSKY, Aaron. «Building sex. Men, women, architecture, and the construction of sexuality». USA, 1995.

BOYER, M. Christine. «City of collective memory». London, The MIT Press. 1996.

ColominA, Beatriz. «Cybercities». New York, Princeton Architectural Press. 1996.

ColominA, Beatriz. «Sexuality \& Space». Princeton Papers on Architecture. Princeton University School of Architecture, New Jersey. 1992.

Colomina, Beatriz. Editor «Privacy and publicity. Modern Architecture as mass media». The MIT Press. London, 1996.

DERRIDA, Jacques. «Intoducción a 'El origen de la geometría de Husserl». Buenos Aires, Ediciones Manantial.pp. 12, 108, 176 y 188. 2.000.

GALLI, Carlo. «La humanidad Multicultural». Madrid, Katz Editores Págs 22, 44 y 64. 2010.

HANKS, William F: «Referential practice». Chicago University Press. Chicago, 1990.

HARVEY, David. «El nuevo imperialismo: acumulación por desposesión» (2004); Pantich, Leo y Colin Leys (ed.) «El Nuevo desafí Imperia»l: 99-129. Buenos Aires: Merlin Press - Clacso. 2004.

HAYDEN, Dolores: «La felicidad entre cuatro paredes». (I'll buy that dream) Arquitectura y vivienda, nº 12, Págs. 34-37. 1987.

De entre todos ellos emergió una arquitectura: un sistema de recursos distribuidos horizontalmente, introducidos como servicios virtuales de TI escalados masivamente y manejados como recursos configurados y mancomunados de manera continua. Este modelo de arquitectura fue inmortalizado por George Gilder en su artículo de octubre 2006 en la revista Wired titulado Las fábricas de información. 
INNERARITY, Daniel. «El nuevo espacio público». Madrid, Espasa. 2006.

PUIG, Toni. «Marca Ciudad. Cómo rediseñarla para asegurar un futuro espléndido para todos». Barcelona. Paidós. pp. 253, 271. 2009.

RÜELI, Katerina y otras. «Designing Practices. Architecture, gender and interdisciplinarity». Black dog Publishing Ld, 1996.

SlOterdiJK, Peter. «Esferas I, II y III».«Esferas I», «Esferas II» y «Esferas III». Madrid, Ediciones Siruela S.A. 2003, 2004 y 2006, respectivamente.

SOKAL, Alan. «Más allá de las imposturas intelectuales. Ciencia, filosofía y cultura». Barcelona, Paidós. Transiciones. Págs 44, 45 y siguientes. 2009.

STEVE, Pile. «The body and the city». Routledge. London and New York, 1996.

Feminismo/s 17, junio 2011, pp. 65-89 